University of Nebraska - Lincoln

DigitalCommons@University of Nebraska - Lincoln

2013

Intense terahertz pulses from SLAC electron beams using coherent transition radiation

Ziran Wu

Stanford University

Alan S. Fisher

Stanford University, afisher@slac.stanford.edu

John Goodfellow

Stanford University

Matthias Fuchs

University of Nebraska - Lincoln, mfuchs@unl.edu

Dan Daranciang

Stanford University, daranciang@stanford.edu

See next page for additional authors

Follow this and additional works at: https://digitalcommons.unl.edu/physicsfuchs

Wu, Ziran; Fisher, Alan S.; Goodfellow, John; Fuchs, Matthias; Daranciang, Dan; Hogan, Mark; Loos, Henrik; and Lindenberg, Aaron, "Intense terahertz pulses from SLAC electron beams using coherent transition radiation" (2013). Matthias Fuchs Publications. 11.

https://digitalcommons.unl.edu/physicsfuchs/11

This Article is brought to you for free and open access by the Research Papers in Physics and Astronomy at DigitalCommons@University of Nebraska - Lincoln. It has been accepted for inclusion in Matthias Fuchs Publications by an authorized administrator of DigitalCommons@University of Nebraska - Lincoln. 


\section{Authors}

Ziran Wu, Alan S. Fisher, John Goodfellow, Matthias Fuchs, Dan Daranciang, Mark Hogan, Henrik Loos, and Aaron Lindenberg 


\section{AIP $\mid \begin{aligned} & \text { Review of } \\ & \text { Scientific Instruments }\end{aligned}$}

Intense terahertz pulses from SLAC electron beams using coherent transition radiation

Ziran Wu, Alan S. Fisher, John Goodfellow, Matthias Fuchs, Dan Daranciang et al.

Citation: Rev. Sci. Instrum. 84, 022701 (2013); doi: 10.1063/1.4790427

View online: http://dx.doi.org/10.1063/1.4790427

View Table of Contents: http://rsi.aip.org/resource/1/RSINAK/v84/i2

Published by the American Institute of Physics.

Additional information on Rev. Sci. Instrum.

Journal Homepage: http://rsi.aip.org

Journal Information: http://rsi.aip.org/about/about_the_journal

Top downloads: http://rsi.aip.org/features/most_downloaded

Information for Authors: http://rsi.aip.org/authors

\section{ADVERTISEMENT}
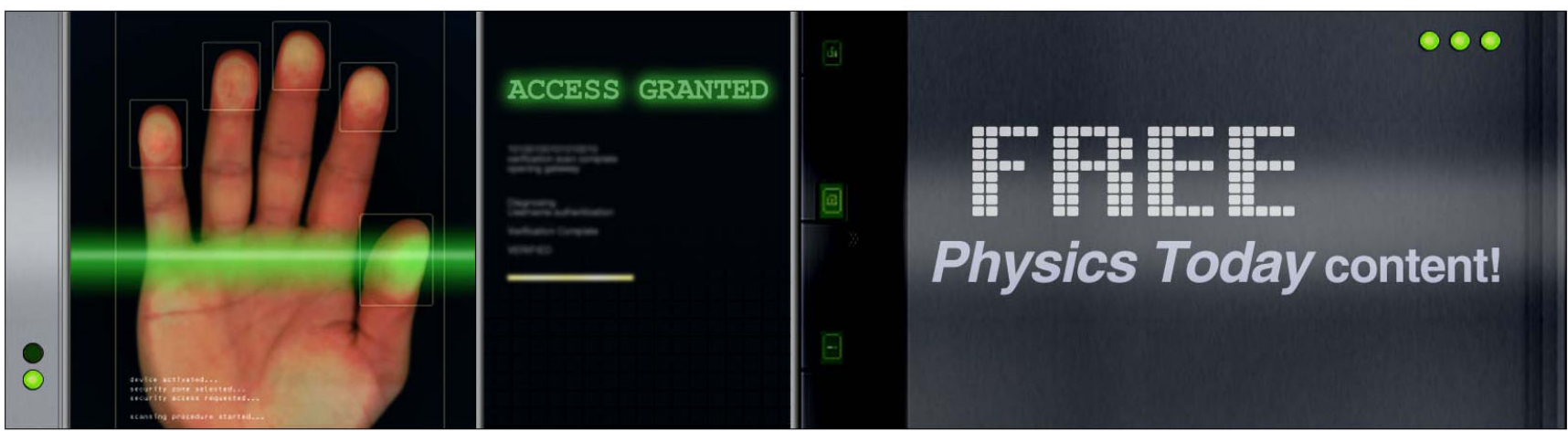


\title{
Intense terahertz pulses from SLAC electron beams using coherent transition radiation
}

\author{
Ziran Wu, ${ }^{1}$ Alan S. Fisher, ${ }^{1, a)}$ John Goodfellow, ${ }^{2,3}$ Matthias Fuchs, ${ }^{3,4}$ Dan Daranciang, ${ }^{5}$ \\ Mark Hogan, ${ }^{1}$ Henrik Loos, ${ }^{1}$ and Aaron Lindenberg ${ }^{2,3,4}$ \\ ${ }^{1}$ Accelerator Directorate, SLAC National Accelerator Laboratory, Stanford University, 2575 Sand Hill Road, \\ Menlo Park, California 94025, USA \\ ${ }^{2}$ Department of Materials Science and Engineering, Stanford University, Stanford, California 94305, USA \\ ${ }^{3}$ Stanford Institute for Materials and Energy Science, SLAC National Accelerator Laboratory, Menlo Park, \\ California 94025, USA \\ ${ }^{4}$ PULSE Institute for Ultrafast Energy Science, SLAC National Accelerator Laboratory, Menlo Park, \\ California 94025, USA \\ ${ }^{5}$ Department of Chemistry, Stanford University, Stanford, California 94305, USA
}

(Received 10 October 2012; accepted 31 December 2012; published online 22 February 2013)

SLAC has two electron accelerators, the Linac Coherent Light Source (LCLS) and the Facility for Advanced Accelerator Experimental Tests (FACET), providing high-charge, high-peak-current, femtosecond electron bunches. These characteristics are ideal for generating intense broadband terahertz (THz) pulses via coherent transition radiation. For LCLS and FACET respectively, the THz pulse duration is typically 20 and 80 fs RMS and can be tuned via the electron bunch duration; emission spectra span 3-30 THz and $0.5 \mathrm{THz}-5 \mathrm{THz}$; and the energy in a quasi-half-cycle THz pulse is 0.2 and $0.6 \mathrm{~mJ}$. The peak electric field at a THz focus has reached $4.4 \mathrm{GV} / \mathrm{m}(0.44 \mathrm{~V} / \AA ̊ \AA)$ at LCLS. This paper presents measurements of the terahertz pulses and preliminary observations of nonlinear materials response. (C) 2013 American Institute of Physics. [http://dx.doi.org/10.1063/1.4790427]

\section{INTRODUCTION}

The terahertz $(\mathrm{THz})$ region of the electromagnetic spectrum (roughly from $100 \mathrm{GHz}$ to $10 \mathrm{THz}$ ) has long been used as a characterization tool, but in recent years the development of new, higher intensity sources, driven by both lasers and electron beams, has created broad interest in using THz light for chemical and biological imaging, and opened up a new regime in which $\mathrm{THz}$ fields can be used to drive nonlinear responses in matter. Other articles in this Special Edition discuss a range of sources and applications.

Spectroscopic applications prefer a source with a narrow frequency span: a long pulse or a continuous source that is tunable over the $\mathrm{THz}$ band. Time-resolved applications prefer short pulses in the single-cycle limit, with correspondingly wide bandwidths, to quickly "kick" a system into an excited state; after a delay of femtoseconds to picoseconds, the response is probed with, for example, an optical pulse from a laser.

A relativistic electron bunch in a linear accelerator (linac) can be compressed during acceleration to a length as short as a few fs, depending on the bunch charge. For wavelengths longer than the bunch length, the emitted fields of all the electrons in the bunch add coherently, and the total radiated power scales with the square of the number of electrons. The electron bunches described here have charges of 100-3000 pC compressed to durations of 10-200 fs RMS, and so are well suited for the generation of intense $\mathrm{THz}$

a) Author to whom correspondence should be addressed. Electronic mail: afisher@slac.stanford.edu. pulses. The electromagnetic fields traveling with the beam can be converted to radiating fields in several ways: by bending the beam in a strong magnetic field (coherent synchrotron radiation and coherent edge radiation, CSR and CER), by passing the beam through a small aperture (coherent diffraction radiation, CDR), or by passing the beam from vacuum into a medium (coherent transition radiation, CTR). Several accelerator-based sources of $\mathrm{THz}$ radiation using these approaches have been constructed. ${ }^{1-11}$

In CTR from a thin metallic foil, surface currents that match boundary conditions on the foil produce the fields of a collapsing dipole as the electrons approach, and an expanding dipole as they leave, generating an impulse of dipole radiation on both sides. ${ }^{12}$ The temporal shape has a long-wavelength cutoff due to the finite size of the foil and apertures. Without these low frequencies, the shortest pulse resembles a "quasihalf-cycle," with a high-amplitude central pulse of one polarity and two longer but weaker lobes of opposite polarity before and after. For highly relativistic electrons, the radiation emitted on exit from the foil travels in the beam direction, while the entrance radiation goes in the direction of specular reflection from the foil surface. By tilting the foil at $45^{\circ}$ to the electron beam, this entrance radiation can be quickly extracted.

SLAC National Accelerator Laboratory has two such THz sources, at the Facility for Advanced Accelerator Experimental Tests (FACET) and at the Linac Coherent Light Source (LCLS). The former uses the first $2 \mathrm{~km}$ of the $3-\mathrm{km}$ SLAC linear accelerator, while the latter uses the third $\mathrm{km}$ (Figure 1). Both sources use CTR, which offers a small and well defined source point (unlike CSR) and the absence of a short-wavelength cutoff from the hole in CDR. A multi-GeV 


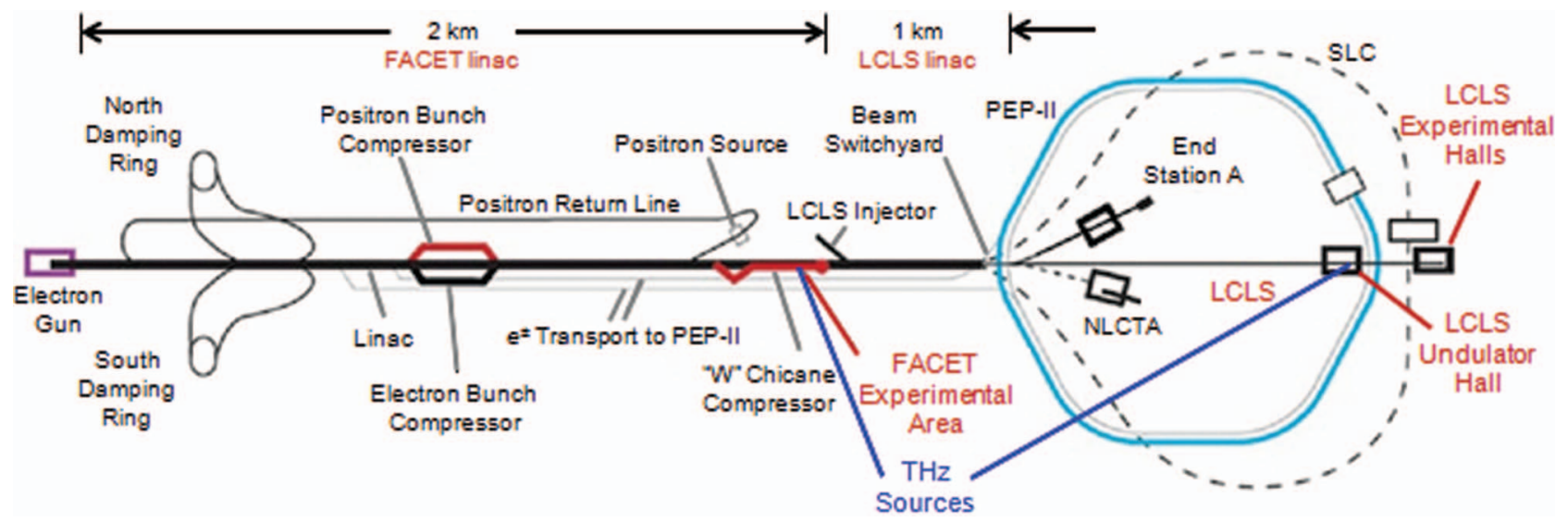

FIG. 1. The 3-km SLAC linac, divided into FACET and LCLS linacs. THz sources are in the FACET experimental area and in the LCLS undulator hall.

beam experiences little scattering when passing through a thin $(\leq 10 \mu \mathrm{m})$ foil.

\section{A. FACET}

The Facility for Advanced Accelerator Experimental Tests $^{13}$ provides 3.2-nC electron (and, in future, positron) bunches at 20-23 GeV, compressed to lengths as short as 67 fs RMS, corresponding to a peak current of over $20 \mathrm{kA}$ (Table I). The beam is tightly focused at the interaction point (IP), a long optical table for user experiments (Figure 2). Advanced acceleration concepts are a major emphasis, notably electron acceleration in wakefields in plasmas ${ }^{14}$ and also in dielectrics. ${ }^{15}$ Both depend on the longitudinal bunch profile, which is measured by a new transverse deflecting cavity and by two THz experiments. The first studies Smith-Purcell radiation. ${ }^{16}$ The second, which is presented here, uses CTR.

\section{B. LCLS}

The Linac Coherent Light Source is the world's first hard x-ray free-electron laser (FEL). ${ }^{17}$ Self-amplified spon-
TABLE I. FACET parameters.

\begin{tabular}{lccc}
\hline \hline & Typical & Best & Design \\
\hline Electron energy $(\mathrm{GeV})$ & 20.35 & 21.1 & 23 \\
Charge per bunch $(\mathrm{pC})$ & $2500-2900$ & 3200 & 3200 \\
Repetition rate $(\mathrm{Hz})$ & 10 & 10 & 30 \\
Bunch length & & & 20 \\
$\sigma_{z}(\mu \mathrm{m})$ & $25-30$ & $<25$ & 67 \\
$\sigma_{t}(\mathrm{fs})$ & $83-100$ & $<83$ & 20 \\
Size at IP & 35 & 20 & 20 \\
$\sigma_{x}(\mu \mathrm{m})$ & 35 & 23 & Double Waist \\
$\sigma_{y}(\mu \mathrm{m})$ & Standard Optics & & 317 \\
& & & 36 \\
\hline Size at upstream & 1200 & & \\
THz foil & 6 & & \\
$\sigma_{x}(\mu \mathrm{m})$ & & & \\
$\sigma_{y}(\mu \mathrm{m})$ & & & \\
\hline \hline
\end{tabular}

taneous emission along more than $100 \mathrm{~m}$ of undulator produces coherent x-ray pulses. The electron energy is tuned over a broad energy range (Table II) to provide a wide range of photon energies, down to wavelengths as short as $1.3 \AA$.

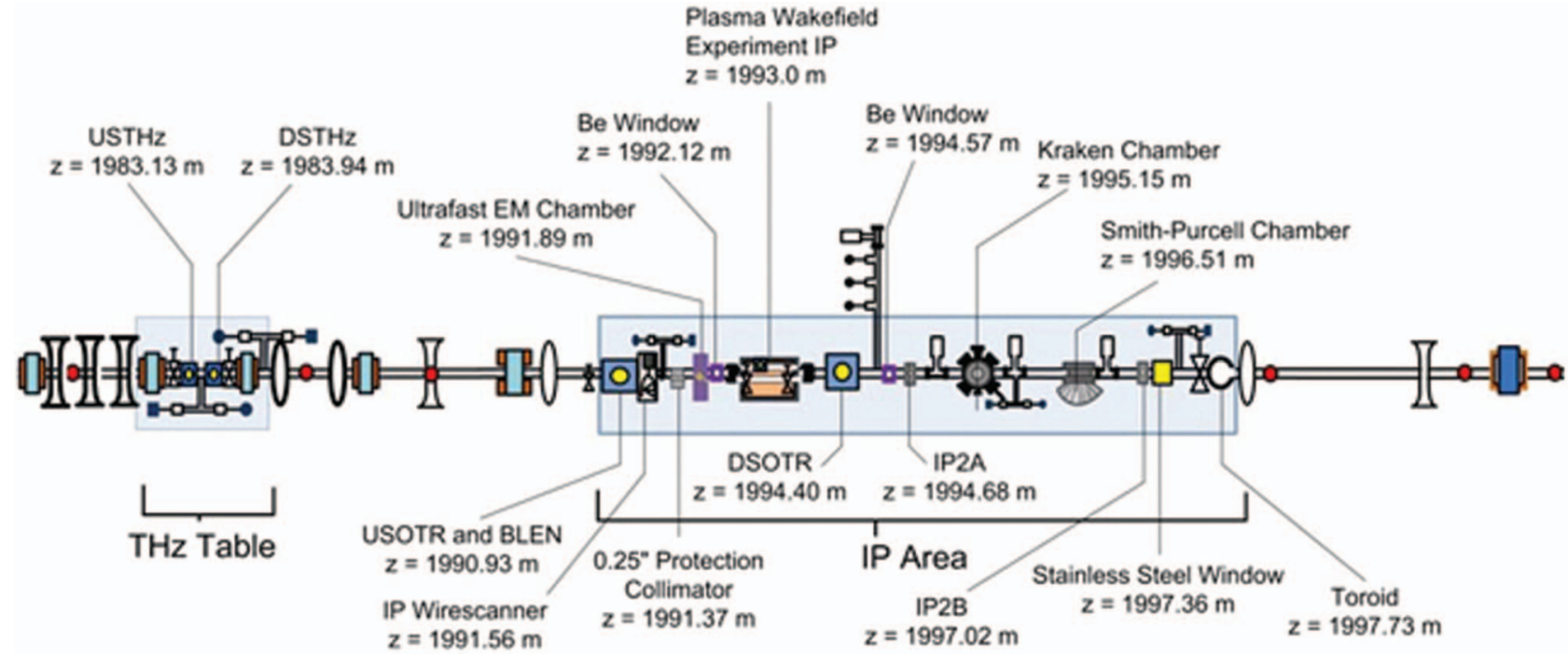

FIG. 2. The THz and IP tables of FACET. 


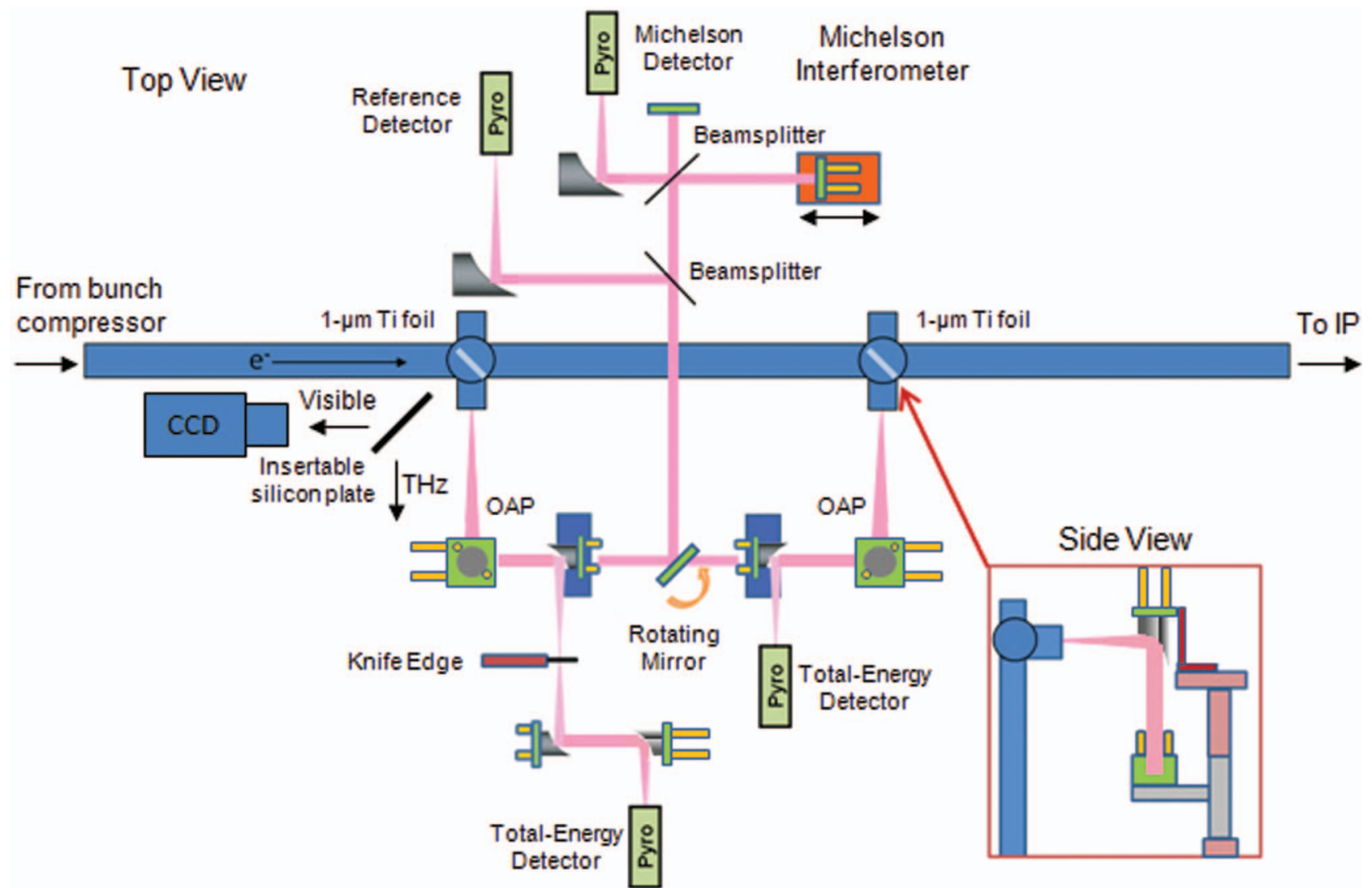

FIG. 3. Layout of the FACET THz table.

Recent progress in x-ray self-seeding ${ }^{18}$ offers nearly Fouriertransform-limited pulses of hard $\mathrm{x}$-rays.

\section{TERAHERTZ AT FACET}

\section{A. Setup}

To provide sufficient space for optics while avoiding interference with experiments on the IP table, the $\mathrm{THz}$ source at FACET is on a separate optical table, $10 \mathrm{~m}$ upstream of the IP (Figure 2). The $\mathrm{THz}$ radiator is a $1-\mu \mathrm{m}$-thick titanium foil rotated about the vertical axis by $45^{\circ}$. As electrons enter the foil, the $\mathrm{THz}$ they emit exits horizontally at

TABLE II. LCLS parameters.

\begin{tabular}{ll}
\hline \hline Electrons & \\
\hline Energy $(\mathrm{GeV})$ & $2.6-14.7$ \\
Charge per bunch $(\mathrm{pC})$ & $20-350$ \\
Repetition rate $(\mathrm{Hz})$ & 120 \\
Bunch length & \\
$\quad \sigma_{z}(\mu \mathrm{m})$ & $1.5-75$ \\
$\quad \sigma_{t}(\mathrm{fs})$ & $5-250$ \\
Size at THz foil & \\
$\quad \sigma_{x, y}(\mu \mathrm{m})$ & $50-70$ \\
$\mathrm{X}$ Rays & \\
\hline Photon energy $(\mathrm{eV})$ & $300-9600$ \\
Pulse energy $(\mathrm{mJ})$ & $0.1-3$ \\
\hline \hline
\end{tabular}

$90^{\circ}$ to the electron direction. The radiation passes through a 25 -mm-diameter, $0.25-\mathrm{mm}$-thick, diamond window and is collimated by an off-axis parabolic mirror (OAP). Instruments on the table (Figure 3) characterize the THz pulses. A pyroelectric joulemeter gives the energy, knife-edge scans measure the size at the focus of another OAP, and a Michelson interferometer provides the spectrum. The entire tabletop is enclosed (Figure 4) and continually purged with dry air to avoid the strong absorption of $\mathrm{THz}$ by water vapor.

The THz table is located after the final bunch compressor but in the middle of the sequence of quadrupole magnets

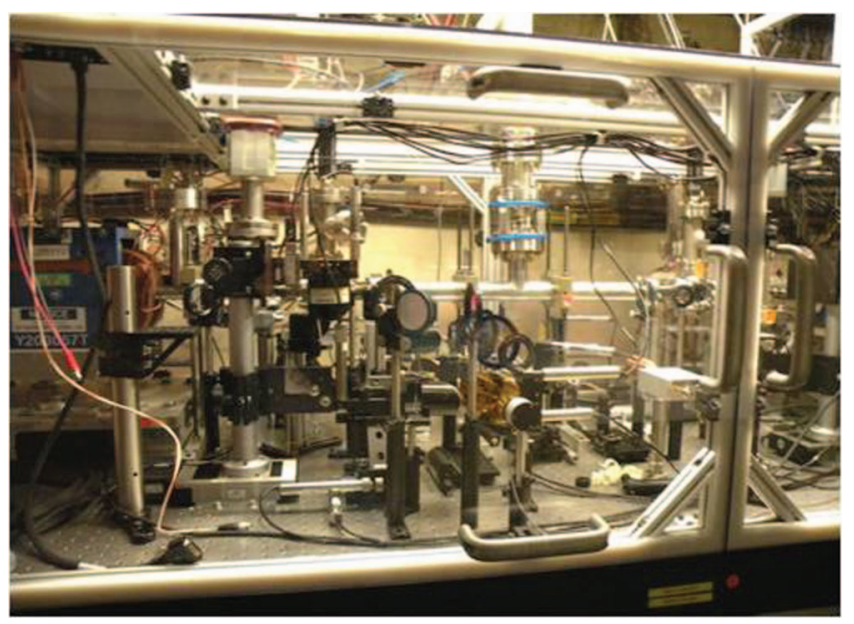

FIG. 4. FACET THz table and dry-air enclosure. 

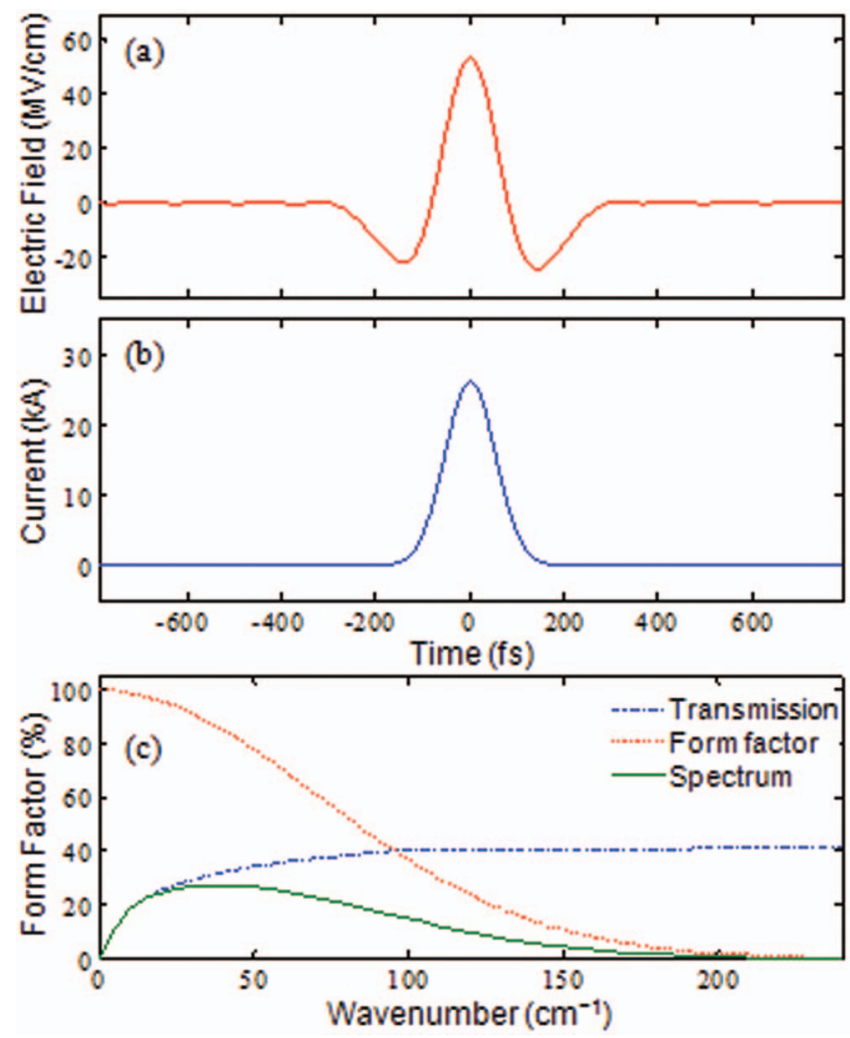

FIG. 5. Calculated (a) CTR electric field, (b) electron-bunch peak current, and (c) CTR power spectrum, using the parameters discussed in the text.

that make the final focus at the IP. With standard IP optics, the nominal beam size at the upstream $\mathrm{THz}$ foil (Table I) is highly elliptical, with $\sigma_{\mathrm{x}}=1.2 \mathrm{~mm}$ and $\sigma_{\mathrm{y}}=6 \mu \mathrm{m}$ at the upstream foil, and $1.5 \mathrm{~mm}$ by $13 \mu \mathrm{m}$ at the downstream foil. The emitted THz energy is reduced for wavelengths shorter than this transverse size, since the fields of the particles interfere destructively. Retuning the quadrupole magnets for a more circular spot at the $\mathrm{THz}$ foil gives a shallow waist of $317 \mu \mathrm{m}$ by $36 \mu \mathrm{m}$ while retaining the small focus at the IP. The measurements reported here use this "double-waist" configuration (Table I).

The $1-\mu \mathrm{m}$ foil thickness is a trade-off between the effect of multiple Coulomb scattering on beam quality and the skin depth, $0.33 \mu \mathrm{m}$ at $1 \mathrm{THz}$, which reduces reflectivity and so the
CTR yield below $1 \mathrm{THz}$. Titanium, a light and strong metal with a high melting temperature, was expected to be resistant to beam damage. However, the beam has punctured several of these foils on the IP table, near the minimum beam radius, and one foil on the $\mathrm{THz}$ table. The damage does not appear to be driven thermally but instead by the peak field, for reasons elaborated in Sec. III A. Other foil materials will be tested in the 2013 FACET run.

\section{B. Simulations}

The THz power and spectrum radiated by CTR was calculated with a code ${ }^{19,20}$ developed at SLAC. The foil is modeled as a perfectly conducting boundary with a unity reflection coefficient. Figure 5 plots the calculated electric field of a 3-nC electron bunch, at an energy of $23 \mathrm{GeV}$, with a 50fs length and a $10-\mu \mathrm{m}$ diameter. For this case, the peak current is roughly $27 \mathrm{kA}$ and the maximum electric field reaches $0.6 \mathrm{~V} / \AA ̊ \AA$ in the $\sim 150 \mathrm{fs}$ long quasi-half cycle pulse. The power spectrum peaks at $1.2 \mathrm{THz}\left(40 \mathrm{~cm}^{-1}\right)$, with significant content up to $6 \mathrm{THz}\left(200 \mathrm{~cm}^{-1}\right)$. The energy per pulse is $13 \mathrm{~mJ}$. The bunch dimensions used were significantly smaller than those available later in the experiment, but the high fields and pulse energy show FACET's potential as a THz source.

Because the experiment initially used the standard optics of Table I, with a ribbon-like beam, another simulation studied the effect on the CTR of the ribbon compared to a circular beam. This required a different code ${ }^{21}$ that follows TerMikaelian. ${ }^{12}$ Figure 6 shows the CTR radiation spectrum, normalized to the square of the charge and integrated over solid angle. The dashed curve corresponds to the elliptical spot of standard optics (Table I, $\sigma_{\mathrm{x}}=1.2 \mathrm{~mm}, \sigma_{\mathrm{y}}=6 \mu \mathrm{m}$ ), the solid line to the double waist $\left(\sigma_{\mathrm{x}}=317 \mu \mathrm{m}, \sigma_{\mathrm{y}}=36 \mu \mathrm{m}\right)$, and the dotted curve to an $85-\mu \mathrm{m}$ circular beam. With double-waist optics, the power at frequencies near $0.5 \mathrm{THz}$ increases by more than a factor of 2 , and the circular beam provides an even greater improvement.

\section{Measurements}

Figure 7(a) shows a typical Michelson interferogram of THz emitted by a highly compressed FACET electron bunch. The pyroelectric detector readings are averaged over 10 shots

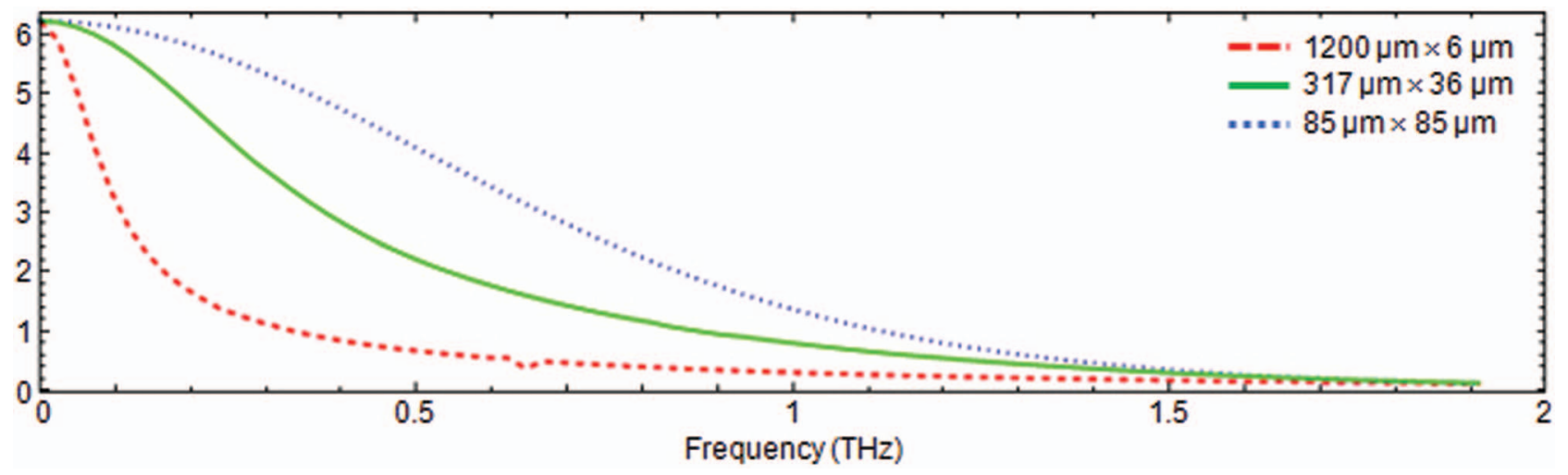

FIG. 6. Calculations comparing the spectrum of the ribbon beam of standard optics (dashed line) to the double waist (solid) and to an $85-\mu \mathrm{m}$ circular beam (dotted). 

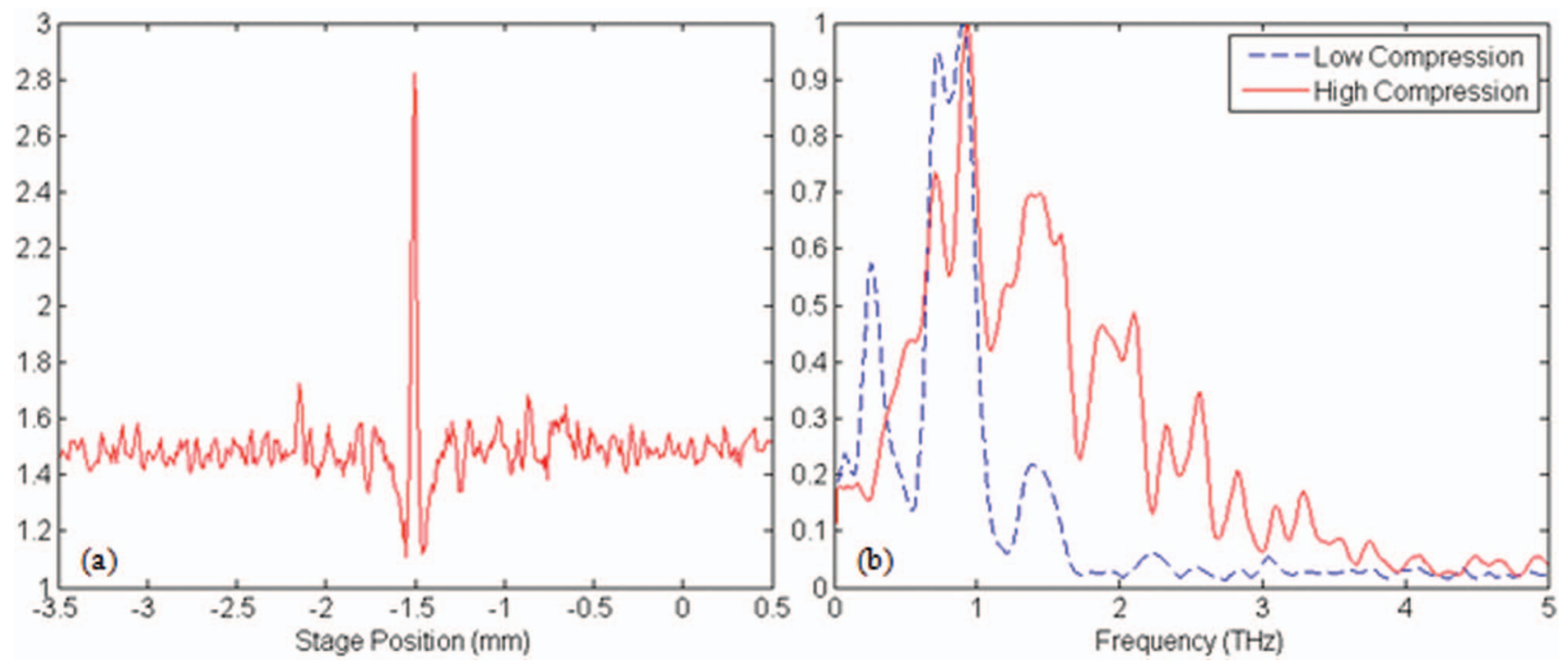

FIG. 7. (a) Michelson interferogram of a CTR THz pulse from a highly compressed electron bunch. The points are normalized to the reference detector. (b) Normalized power spectra of THz pulses from bunches with low and high compression.

and normalized to readings from a reference pyro detector right before the interferometer (Figure 3). The 4-mm scan has a resolution of $37 \mathrm{GHz}$ in the frequency domain.

Fourier transforms of the scans give power spectra. Figure 7(b) shows the change in spectral content for high and low bunch compression. Strong water absorption lines near $1.3 \mathrm{THz}$ are not present, due to the low humidity inside the enclosure. We attribute the $0.24-\mathrm{THz}$ modulation in both spectra to Fabry-Pérot interference in reflections from the layers of the pyroelectric detector. The spectra were smoothed to reduce this effect.

The spectrum can be used to reconstruct the temporal profile of a bunch. Although the wakefield of a relativistic bunch follows the bunch shape, the fields radiated by the foil are filtered by the finite beampipe and window, and so lose long wavelengths. To compensate the spectrum for this loss, we model the attenuation of the field as a Gaussian highpass filter, ${ }^{22} 1-\exp \left(-f^{2} / f_{0}{ }^{2}\right)$, which leads to negative lobes on either side of the main peak of the interferogram. With data from a highly compressed beam, which has a nearly Gaussian profile, we fit the center of the autocorrelation in the time domain (Figure 8(a)) with a Gaussian beam and filter to find the filter constant $f_{0}$. For FACET, $f_{0}$ lies between 0.35 and $0.45 \mathrm{THz}$, depending on the adjustment of the optics. We then discard the assumption of a Gaussian beam but retain $f_{0}$. The power spectrum is compensated by dividing by the square of the filter down to frequencies near $f_{0}$, where the correction starts to diverge and where little signal is available. From this point down to zero frequency, the correction is completed by fitting a Gaussian peaking at $f=0$ (Figure 8(b)). Empirically, it is found that the reconstruction does not depend strongly on the detailed shape at the
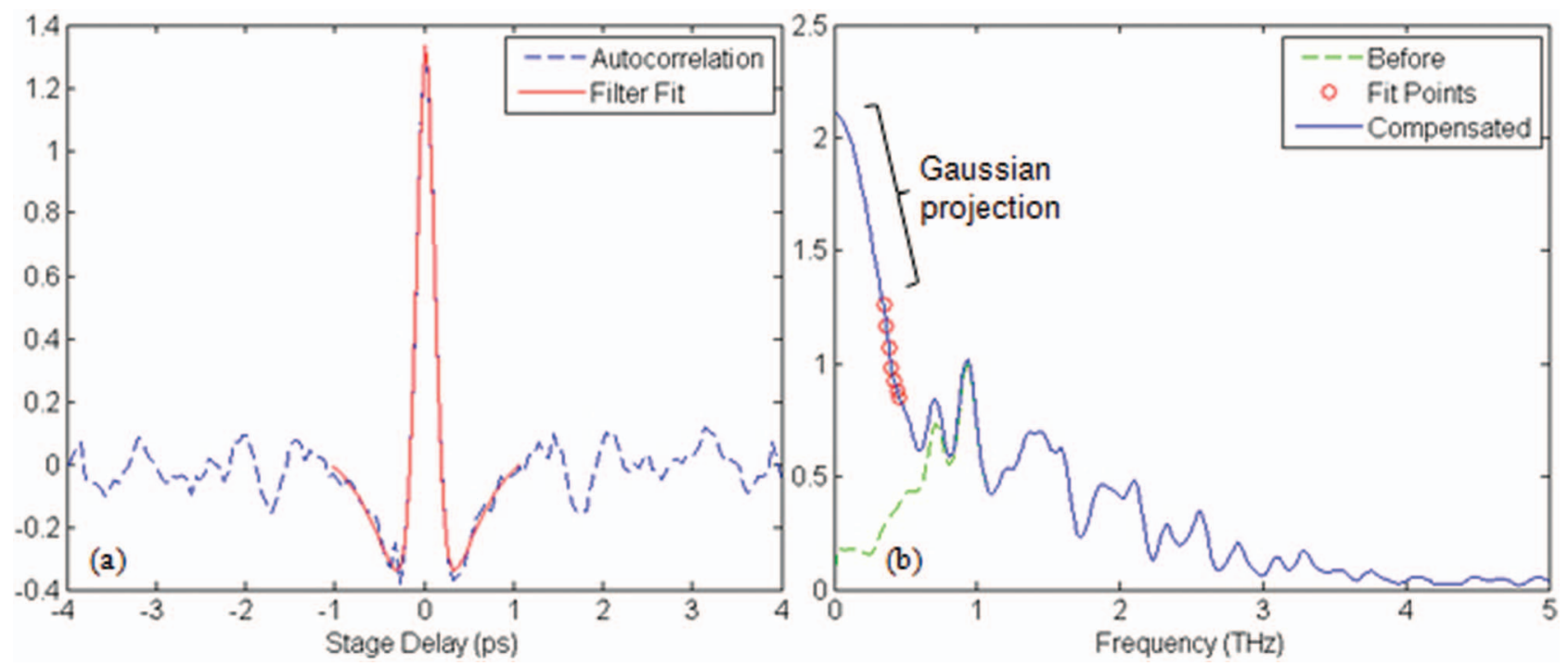

FIG. 8. (a) Central region of the autocorrelation of Figure 7(a), after subtracting the mean, with a fit about the peak to find the high-pass filter. (b) The highcompression spectrum (dashed line) of Figure 7(b) is compensated (solid) for the high-pass filter, down to the leftmost circled point, below which a Gaussian fit to all the circled points extends the curve to $f=0$. 

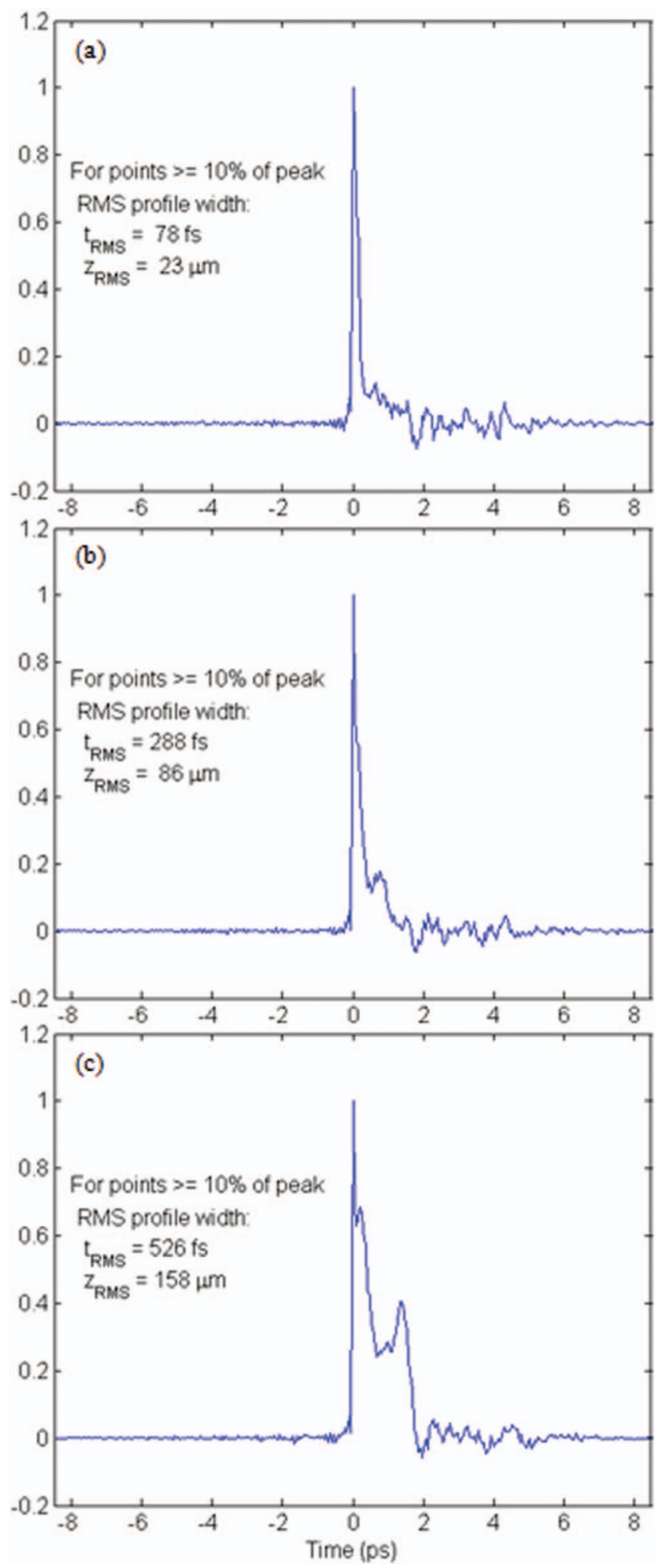

FIG. 9. Reconstruction of bunches at (a) high, (b) medium, and (c) low compression. A typical "double horn" structure develops as the compression is reduced.

lowest frequencies. Then the Kramers-Kronig relations allow reconstruction of the electron-bunch profile from the corrected spectrum, ${ }^{23}$ as shown in Figure 9.

If this spectral correction is not applied, the KramersKronig procedure reconstructs the electric field of the $\mathrm{THz}$ pulse on the optical table. Figure 10 shows an example us-

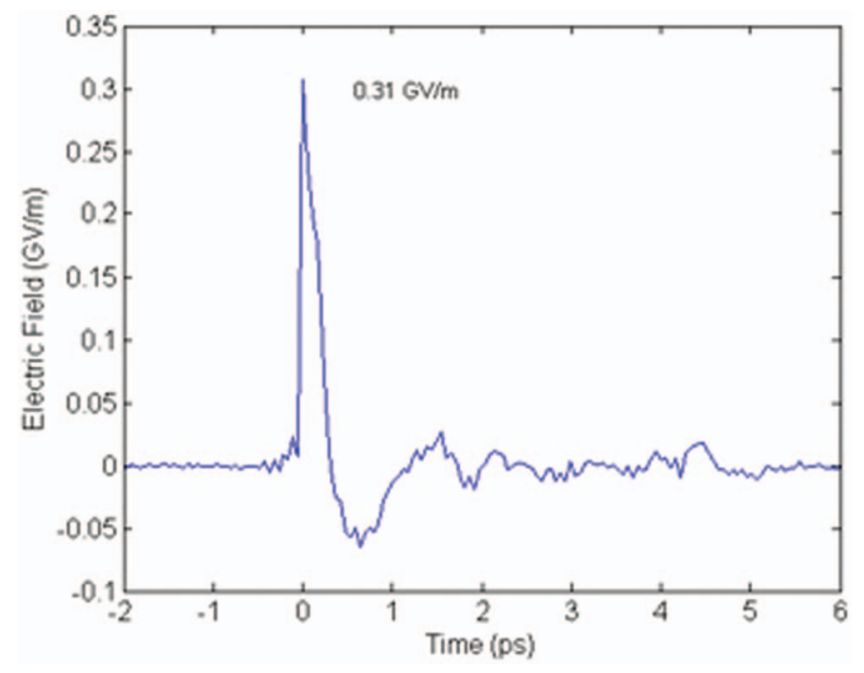

FIG. 10. Reconstruction of the focused THz electric field in FACET.

ing intermediate compression, in which measurements of the pulse energy $(350 \mu \mathrm{J})$ and the transverse beam sizes $(1.36$ and $1.09 \mathrm{~mm}$ RMS, using knife-edge scans across a THz focus) were made along with the autocorrelation. The calculation gives a field peaking at $0.31 \mathrm{GV} / \mathrm{m}$, corresponding to a magnetic field of $1.0 \mathrm{~T}$. A reconstruction of this bunch gives an RMS length of $130 \mathrm{fs}$, double the length of the shortest FACET bunches. These should yield twice the energy and field.

The Kramers-Kronig algorithm does not resolve time reversal and generally places the main peak first; moreover, the entire tail usually appears afterward, even for a symmetric single-cycle pulse like that in Figure 5(a). However, the bunch asymmetry in Fig. 9 agrees well with measurements (Figure 11) using a newly commissioned X-band transverse deflecting cavity (TCAV). ${ }^{24}$

With the double-waist electron optics discussed in Secs. II A and II B and in Table I, a shallow waist with a

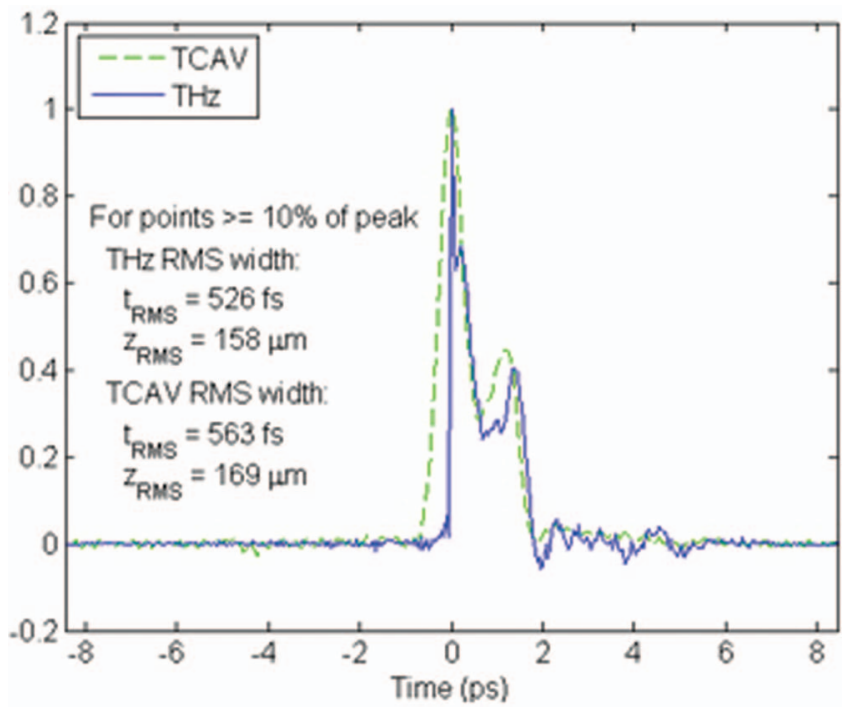

FIG. 11. A comparison of the THz profile reconstruction of Figure 9(c) with a measurement using a transverse deflecting cavity. 

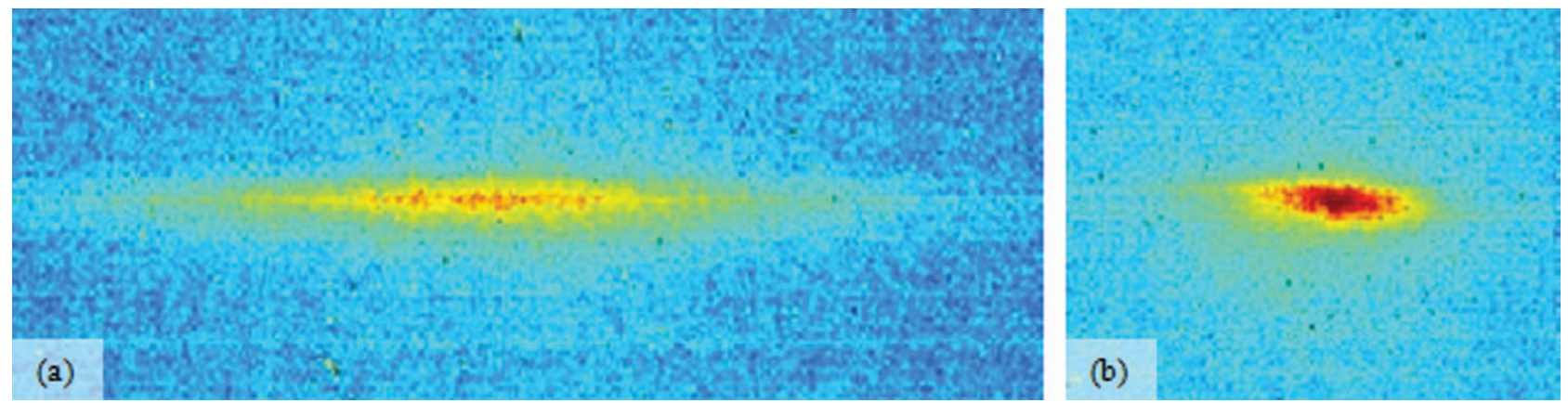

FIG. 12. The FACET electron beam on the upstream THz foil, imaged with the same scale using optical transition radiation, for (a) standard and (b) double-waist electron optics.

more circular electron beam is formed at the upstream $\mathrm{THz}$ foil. Figure 12 compares optical-transition-radiation images of the transverse spot on the foil with the standard and doublewaist configurations; the size scales as expected. The THz energy per pulse increased by $33 \%$, from 0.47 to $0.63 \mathrm{~mJ}$, and the spectrum shows more high-frequency content in qualitative agreement with Figure 6. Quadrupole settings have been calculated to produce even smaller sizes, including the circular spot of Figure 6, when the constraint of a small size at the IP is lifted. Dedicated beam time to test these optical configurations has been requested during the 2013 run in order to determine the largest transverse size consistent with maximum CTR.

\section{TERAHERTZ AT LCLS}

\section{A. Setup}

The LCLS THz source is located in the Undulator Hall, $31 \mathrm{~m}$ downstream of the undulator. Measurements have been carried out at electron energies of $14 \mathrm{GeV}$ with bunch charges from $150-350 \mathrm{pC}$. A beryllium foil tilted downward by $45^{\circ}$ sends $\mathrm{THz}$ through a 25 -mm-diameter, 0.25 -mm-thick diamond window to optics on a table below. These include characterization measurements similar to those at FACET, plus a 20-fs Ti:sapphire oscillator, for THz-pump and laser-probe studies. The reflection and transmission of a variety of samples can be measured as they are exposed to intense $\mathrm{THz}$ pulses. Again the tabletop is enclosed, both for a dry-air purge and for laser safety.

Initial measurements used a $2-\mu \mathrm{m}$-thick beryllium foil, a metal chosen both for low scattering of the electron beam and for weak absorption of FEL $\mathrm{x}$ rays that also pass through the beampipe. The skin depth for Be is $95 \mathrm{~nm}$ at $1 \mathrm{THz}$. This foil was broken after several months when a vacuum-valve controller malfunctioned. To avoid the toxicity of Be particulates, aluminum was substituted. Maintaining low x-ray absorption required a 500-nm thickness, which is still much larger than the 85-nm skin depth at $1 \mathrm{THz}$.

The electron beam punctured three $\mathrm{Al}$ foils, even when the undulators were pulled away from the beam so that no FEL $x$ rays were produced. In a systematic study with a constant charge of $150 \mathrm{pC}$ and a low $10-\mathrm{Hz}$ repetition rate, the bunch was gradually compressed. No damage was seen un- til the bunch length was reduced to 100 fs FWHM. Then a video camera detected light emitted from the beam position on the foil, and within seconds a small hole $(\sim 1 \mathrm{~mm})$ was visible. We concluded that damage depending on compression at the femtosecond timescale cannot be thermal but is likely a mechanism driven by the peak field of the bunch, such as a surface discharge. Material selection by thermal and mechanical properties is not sufficient. We resumed work with Be, but with a $10-\mu \mathrm{m}$-thickness and a new holder that parks the Be when not in use inside a volume with low conductance to the beampipe. This design slows a sudden pressure rise and equalizes the pressure on both sides of the foil. Be has proven to be resistant to beam damage, even at full charge ( $350 \mathrm{pC})$ and compression (50 fs FHWM).

\section{B. Simulations}

Simulations of the radiated field strength and temporal profile are similar to those described above for FACET. The spatio-temporal electron beam profile is obtained by a simulation of the entire LCLS accelerator using Impact-T and Elegant codes. ${ }^{25}$ The initial electric field at the Be foil is transformed into a frequency-domain profile and propagated using Laguerre-Gaussian modes through the optical setup, including diffraction effects from the finite size of the foil. These simulations calculate the full extracted spatio-temporal $\mathrm{THz}$ field at the focus of the optical setup, predicting a radially polarized beam with zero intensity on axis, reflecting the relativistic Coulomb field of the electron bunch. For the highest charge and shortest bunch measured ( $350 \mathrm{pC}, 50 \mathrm{fs}$ FWHM), at the focus of an OAP with an effective focal length of $150 \mathrm{~mm}(\mathrm{NA}=0.1)$, the model predicts a $400-\mu \mathrm{m}$ FWHM transverse profile and a peak field $100 \mu \mathrm{m}$ from the axis of $7 \mathrm{GV} / \mathrm{m}(0.7 \mathrm{~V} / \AA ̊ \AA)$. Integrating over the temporal and spatial profile gives a radiated energy of $560 \mu \mathrm{J}$.

\section{Measurements}

Total energy measurements of the radiated $\mathrm{THz}$ fields are carried out using several methods to check for consistency. A thermopile power meter with a graphite absorber and a pyroelectric detector (Gentec) were used initially, with maximum recorded energy of $\sim 200 \mu \mathrm{J} /$ pulse. The energy may be higher, since Gentec later provided preliminary calibration correction 


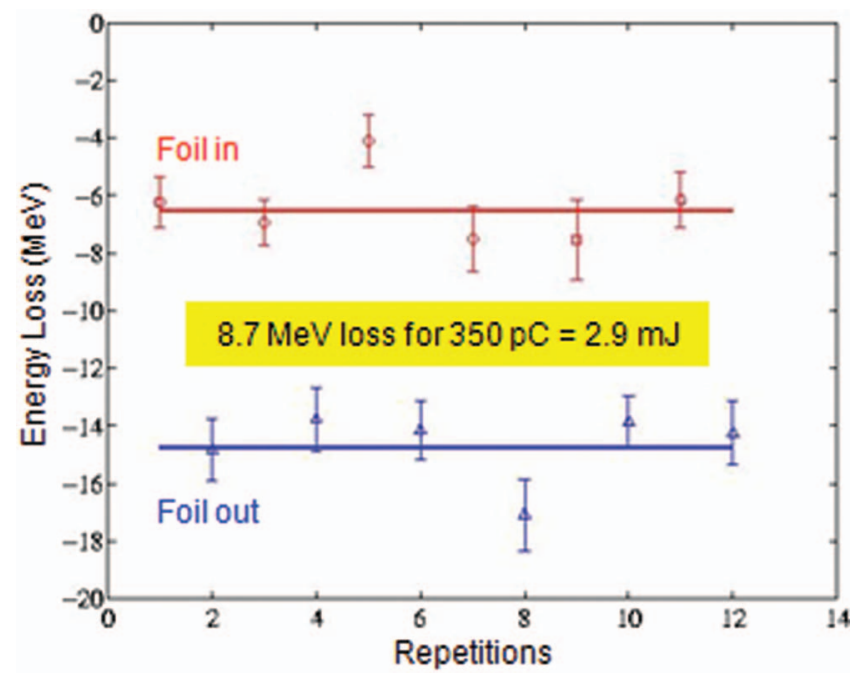

FIG. 13. Electron-beam energy loss due to inserting the $2-\mu \mathrm{m}$ Be foil.

factors of 2.1-4 at $2.52 \mathrm{THz}$, depending on the detector type. Similarly, the graphite in the thermopile is not a perfect $\mathrm{THz}$ absorber.

The total radiated $\mathrm{THz}$ energy can also be obtained by measuring the energy loss of the electrons due to CTR, an approach not subject to uncertainties about the broadband calibration of the detectors. The bend at the electron beam dump was designed as an energy spectrometer, allowing us to compare the deflection with the foil radiator in and out of the beam. The electron beam is estimated to lose only $30 \mu \mathrm{J}$ in a $2-\mu \mathrm{m}$ Be foil tilted at $45^{\circ}$, and $150 \mu \mathrm{J}$ in a $10-\mu \mathrm{m}$ foil. ${ }^{26}$ Figure 13 shows measurements with the $2-\mu \mathrm{m}$ Be foil indicating an $8.7-\mathrm{MeV}$ loss per electron, corresponding to a 2.9-
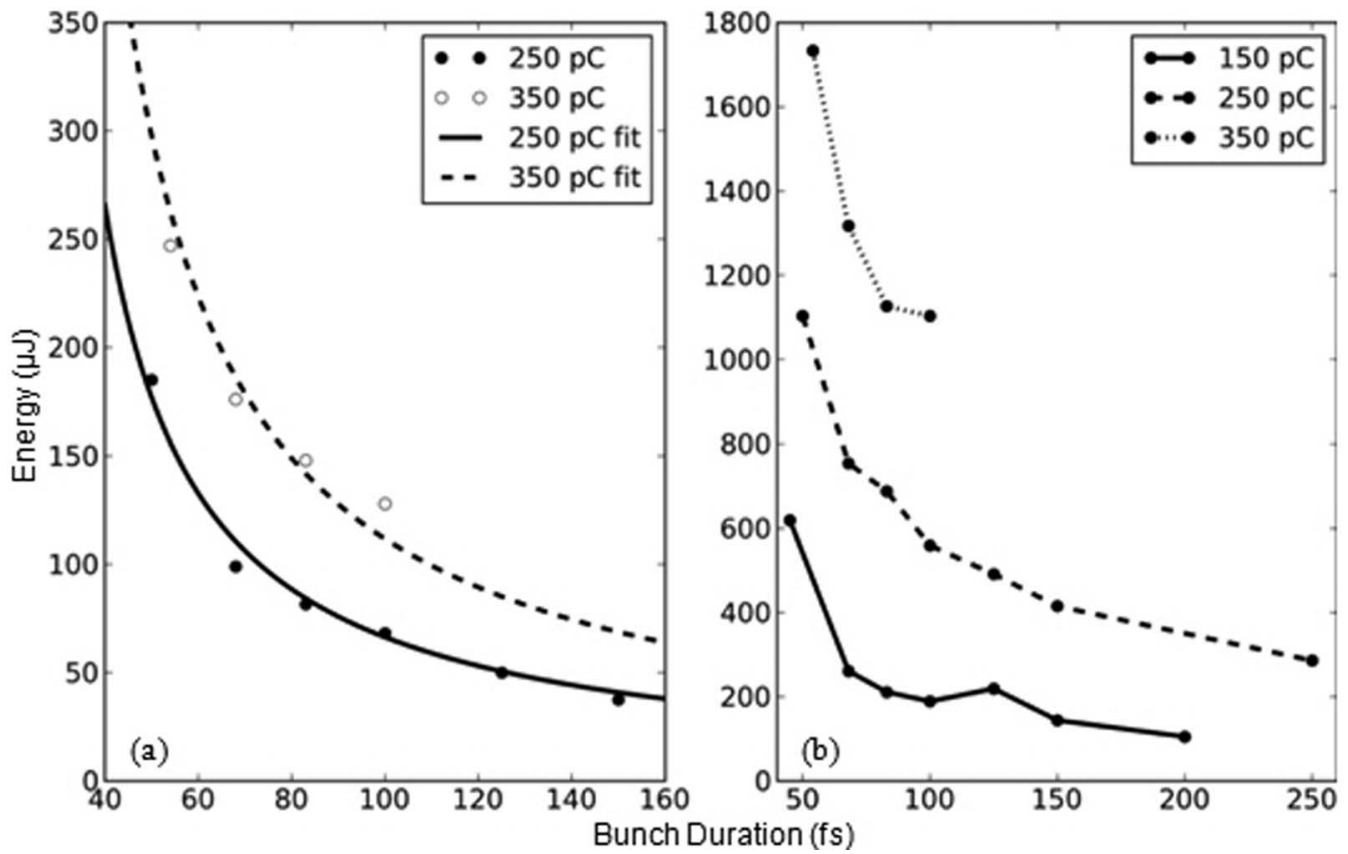

FIG. 14. Dependence of THz energy on charge and FWHM bunch duration, measured by (a) a pyroelectric joulemeter at a THz focus, and (b) the electron-beam energy loss.

$\mathrm{mJ}$ loss for $350 \mathrm{pC}$. Half of this $\mathrm{THz}$ is radiated in the forward direction as the beam exits the foil. The radiation on entrance heads downward, but $30 \%$ is lost by reflection from the high-index diamond window, so that about $1 \mathrm{~mJ}$ would be expected on the table. Additional losses, particularly absorption by water vapor (before the enclosure was installed) reduce these fields by another factor of $\sim 2$. This approach provides a complimentary estimate of the radiated fields. Given the uncertainties, the simulations and the energy loss are in reasonable agreement with the detector measurements.

For a charge $q$ and pulse duration $t$, the radiated $\mathrm{THz}$ field is expected to scale with the time-dependent peak current $q / t$, and the intensity with $q^{2} / t^{2}$. Integrating over time, the radiated energy then scales with $q^{2} / t$. Figure 14 shows measurements which roughly confirm this scaling law, using (a) a pyroelectric detector at a $\mathrm{THz}$ focus, and (b) the electron energy loss. This scaling cannot be precise: the bunch shape changes with compression, becoming more peaked and increasing emission faster than $1 / t$. Also, the nominal bunch length in the figure was taken from a monitor measuring filtered infrared from a bend at the end of the final compressor. It was calibrated with a transverse deflecting cavity, but the bandpass filter makes it somewhat inaccurate for the shortest bunches. The best fit to the above scaling offsets the bunch length by approximately 20 fs.

As with FACET, the Kramers-Kronig procedure can reconstruct the temporal profile of the electric field from an autocorrelation. Using a measured autocorrelation of a 350-pC, 70-fs FWHM bunch and a typical energy of $180 \mu \mathrm{J}$ from Figure 14, the reconstruction in Figure 15 calculates a peak field of $4.4 \mathrm{GV} / \mathrm{m}$ at the focus on the optical table. This field is much stronger than the FACET field in Figure 10 since this energy is contained in a shorter bunch and a smaller focal spot, $200 \mu \mathrm{m}$ RMS. The smaller source size and shorter bunch 


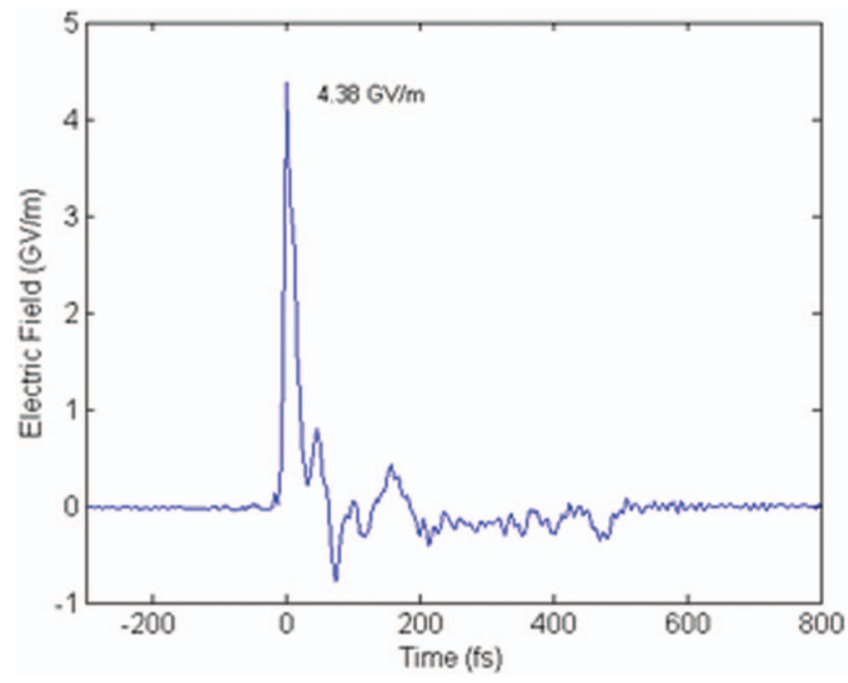

FIG. 15. Reconstruction of the focused THz field in LCLS.

result in a spectrum with higher frequencies, peaking at 10 $\mathrm{THz},{ }^{11}$ and thus allow a tighter focus.

Further evidence for the high field strength is provided by nonlinear autocorrelations. The field (linear) autocorrelation shown in Figure 7(a) provides a direct measurement of the spectrum of the radiated field. The peak-to-baseline ratio for such scans is limited to 2 , with a perfect overlap of the fields in space and time on the pyroelectric detector. In other autocorrelations, two THz pulses were incident collinearly on a silicon photodiode, which provides an intrinsically nonlinear response since its 1.1-eV bandgap is well above the $\mathrm{THz}$ photon energy. ${ }^{27}$ Figure 16 plots the diode's photocurrent as a function of the relative time delay between the two $\mathrm{THz}$ fields for a bunch with intermediate compression (90 fs FWHM for 350 $\mathrm{pC}$ ). The scan shows the 3-peak autocorrelation expected for a double-horn distribution, ${ }^{11}$ but with a high peak-to-baseline ratio of 9 that indicates the photodiode's strongly nonlinear response to the high fields.

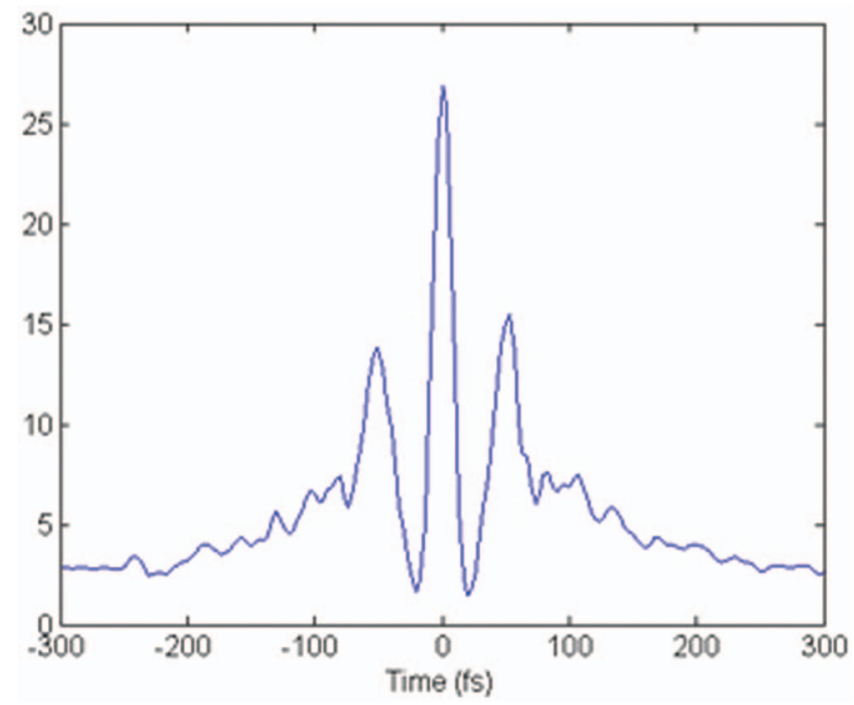

FIG. 16. A nonlinear autocorrelation measured with a silicon photodiode.

\section{ACKNOWLEDGMENTS}

This work was supported by the U.S. Department of Energy under Contract DE-AC02-76SF00515. The Linac Coherent Light Source (LCLS) and at the Facility for Advanced Accelerator Experimental Tests (FACET) are User Facilities at SLAC National Accelerator Laboratory operated for the U.S. Department of Energy, Office of Science, by Stanford University. The authors gratefully acknowledge the support of the DOE Office of Basic Energy Sciences for LCLS and the Office of High-Energy Physics for FACET, and A.L. also acknowledges the support of the Office of Basic Energy Sciences, Materials Sciences and Engineering Division.

M.F. gratefully acknowledges financial support from the Volkswagen Foundation. J.G. is supported in part by the Department of Energy Office of Science Graduate Fellowship Program (DOE SCGF), made possible in part by the American Recovery and Reinvestment Act of 2009, administered by ORISE-ORAU (Contract No. DE-AC05-06OR23100).

${ }^{1}$ G. L. Carr, M. C. Martin, W. R. McKinney, K. Jordan, G. R. Neil, and G. P. Williams, "High-power terahertz radiation from relativistic electrons," Nature 420, 153-156 (2002).

${ }^{2}$ J. van Tilborg, C. B. Schroeder, C. V. Filip, Cs. Tóth, C. G. R. Geddes, G. Fubiani, R. Huber, R. A. Kaindl, E. Esarey, and W. P. Leemans, "Temporal characterization of femtosecond laser-plasma-accelerated electron bunches using terahertz radiation,” Phys. Rev. Lett. 96, 014801 (2006).

${ }^{3}$ M. C. Hoffmann, S. Schulz, S. Wesch, S. Wunderlich, A. Cavalleri, and B. Schmidt, "Coherent single-cycle pulses with MV/cm field strengths from a relativistic transition radiation light source," Opt. Lett. 36, 4473-4475 (2011).

${ }^{4}$ T. Takahashi, Y. Shibata, F. Arai, K. Ishi, T. Ohsaka, M. Ikezawa, Y. Kondo, T. Nakazato, S. Urasawa, and R. Kato, "Coherent transition radiation at submillimeter and millimeter wavelengths," Phys. Rev. E 48, 4674 (1993).

${ }^{5}$ S. Casalbuoni, B. Schmidt, P. Schmüser, V. Arsov, and S. Wesch, "Ultrabroadband terahertz source and beamline based on coherent transition radiation," Phys. Rev. ST Accel. Beams 12, 030705 (2009).

${ }^{6} \mathrm{C}$. S. Thongbai and T. Vilaithong, "Coherent transition radiation from short electron bunches," Nucl. Instrum. Methods A 581, 874 (2007).

${ }^{7}$ D. Mihalcea, C. Bohn, U. Happek, and P. Piot, "Longitudinal electron bunch diagnostics using coherent transition radiation," Phys. Rev. ST Accel. Beams 9, 082801 (2006).

${ }^{8}$ Y. Shen, T. Watanabe, D. A. Arena, C.-C. Kao, J. B. Murphy, T. Y. Tsang, X. J. Wang, and G. L. Carr, "Nonlinear cross-phase modulation with intense single-cycle terahertz pulses," Phys. Rev. Lett. 99, 043901 (2007).

${ }^{9}$ T. Nakazato, M. Oyamada, N. Niimura, S. Urasawa, O. Konno, A. Kagaya, R. Kato, T. Kamiyama, Y. Torizuka, and T. Nanba, "Observation of coherent synchrotron radiation," Phys. Rev. Lett. 63, 1245 (1989).

${ }^{10}$ G. Andonian, A. Cook, M. Dunning, E. Hemsing, G. Marcus, A. Murokh, S. Reiche, D. Schiller, J. B. Rosenzweig, M. Babzien, K. Kusché, and V. Yakimenko, "Observation of coherent terahertz edge radiation from compressed electron beams," Phys. Rev. ST Accel. Beams 12, 030701 (2009).

${ }^{11}$ D. Daranciang, J. Goodfellow, M. Fuchs, H. Wen, S. Ghimire, D. A. Reis, H. Loos, A. S. Fisher, and A. M. Lindenberg, "Single-cycle terahertz pulses with $>0.2$ V/Å field amplitudes via coherent transition radiation," Appl. Phys. Lett. 99, 141117 (2011).

${ }^{12}$ M. L. Ter-Mikaelian, High-Energy Electromagnetic Processes in Condensed Media (Wiley, New York, 1972).

${ }^{13}$ C. I. Clarke, F. J. Decker, R. J. England, R. Erikson, C. Hast, M. J. Hogan, S. Z. Li, M. Litos, Y. Nosochkov, J. Seeman, J. Sheppard, U. Wienands, M. Woodley, and G. Yocky, "FACET: SLAC's New User Facility," SLACPUB-15025, May 2012.

${ }^{14}$ I. Blumenfeld, C. E. Clayton, F.-J Decker, M. J. Hogan, C. Huang, R. Ischebeck, R. Iverson, C. Joshi, T. Katsouleas, N. Kirby, W. Lu, K. A. Marsh, W. B. Mori, P. Muggli, E. Oz, R. H. Siemann, D. Walz, and M. Zhou, "Energy doubling of $42 \mathrm{GeV}$ electrons in a metre-scale plasma wakefield accelerator," Nature 445, 741 (2007). 
${ }^{15}$ G. Andonian, D. Stratakis, M. Babzien, S. Barber, M. Fedurin, E. Hemsing, K. Kusché, P. Muggli, B. O'Shea, X. Wei, O. Williams, V. Yakimenko, and J. B. Rosenzweig, "Dielectric wakefield acceleration of a relativistic electron beam in a slab-symmetric dielectric lined waveguide," Phys. Rev. Lett. 108, 244801 (2012).

${ }^{16}$ V. Blackmore, G. Doucas, B. Ottewell, C. Perry, M. F. Kimmitt, R. Arnold, S. Molloy, and M. Woods, "First measurements of the longitudinal bunch profile at SLAC using coherent Smith-Purcell radiation at $28 \mathrm{GeV}$," SLACPUB-13499, November 2011.

${ }^{17}$ P. Emma et al., "First lasing and operation of an ångstrom-wavelength freeelectron laser," Nature Photon. 4, 641-647 (2010).

${ }^{18} \mathrm{~J}$. Amann et al., "Demonstration of self-seeding in a hard-X-ray freeelectron laser," Nature Photon. 6, 693-698 (2012).

${ }^{19}$ H. Loos, R. Akre, A. Brachmann, F.-J. Decker, Y. Ding, D. Dowell, P. Emma, J. Frisch, S. Gilevich, G. Hays, Ph. Hering, Z. Huang, R. Iverson, C. Limborg-Deprey, A. Miahnahri, S. Molloy, H.-D. Nuhn, J. Turner, J. Welch, W. White, and J. Wu, "Observation of coherent optical transition radiation in the LCLS LINAC," SLAC-PUB-13395, September 2008.

${ }^{20}$ J. D. Jackson, "Collisions, energy loss, and scattering of charged particles,
Cherenkov and transition radiation," in Classical Electrodynamics (Wiley, New York, 1975).

${ }^{21}$ J. England, SLAC, private communication (2011).

${ }^{22}$ A. Murokh, J. B. Rosenzweig, M. Hogan, H. Suk, G. Travish, and U. Happek, "Bunch length measurement of picosecond electron beams from a photoinjector using coherent transition radiation," Nucl. Instrum. Methods A 410, 452 (1998).

${ }^{23}$ R. Lai and A. J. Sievers, "On using the coherent far IR radiation produced by a charged-particle bunch to determine its shape: I Analysis," Nucl. Instrum. Methods A 397, 221 (1997).

${ }^{24}$ M. Litos, SLAC, private communication (2012).

${ }^{25}$ J. Qiang, S. Lidia, R. Ryne, and C. Limborg-Deprey, "Three-dimensional quasistatic model for high brightness beam dynamics simulation," Phys. Rev. ST Accel. Beams 9, 044204 (2006).

${ }^{26}$ A Physicist's Desk Reference, the Physics Vade Mecum, 2nd ed., edited by H. L. Anderson (Springer, 1989), p. 260, sec. 16.07.F.

${ }^{27}$ K. Briggman, L. Richter, and J. Stephenson, "Imaging and autocorrelation of ultrafast infrared laser pulses in the 3-11 micron range with silicon CCD cameras and photodiodes," Opt. Lett. 26, 238 (2001). 